\title{
Avaliação neurológica de recém-nascidos a termo de baixo risco pelo Método Dubowitz
}

\author{
Neurological evaluation of low risk term neonates in \\ Dubowitz Method
}

Kathrein Koike do Nascimento', Gabriela Monika Ay Casagrande',2, Marina Ortega Golin'

\begin{abstract}
Resumo
Introdução: Ao longo dos anos vêm surgindo novas técnicas para avaliar o desenvolvimento dos recém-nascidos a termo. Objetivo: comparar as respostas obtidas na avaliação neurológica neonatal em recém-nascidos a termo (RNT) de baixo risco para lesões neurológicas com as esperadas para a normalidade propostas, pelo método padronizado de Dubowitz. Casuística e Métodos: Foi realizado estudo prospectivo transversal composto por 30 RNT de baixo risco, na maternidade do Hospital Estadual Mário Covas - Santo André (SP). A avaliação neurológica neonatal de Dubowitz foi aplicada em RNT nascidos com idades gestacionais entre $37^{\mathrm{a}}$ e $42^{\mathrm{a}}$ semanas, peso ao nascimento superior ou igual a $2.500 \mathrm{~g}$, sem registros de intercorrências. Para análise dos resultados foram calculados média e desvio padrão, sendo que o nível de significância adotado foi $p<0,05$ para análise comparativa entre as pontuações das avaliações, pelo Teste Qui-quadrado. Resultados: $80 \%$ dos RNTs apresentaram pontuação esperada para a normalidade ( $p<0,001)$. Nenhuma característica da população analisada foi associada às pontuações abaixo do esperado. Conclusões: 0 s resultados aqui encontrados sugerem a possibilidade de aplicação do método Dubowitz na população de RNT devido à similaridade de respostas.
\end{abstract}

Palavras-chave: recém-nascido; desenvolvimento infantil; exame neurológico.

\section{Abstract}

Introduction: Over the years new techniques has emerged to assess the development of newborns Objective: compare the responses obtained from the neonatal neurological assessment in term neonates at low risk for neurological damage to those expected for normality proposed by the standard method of Dubowitz. Casuistry and Methods: there was conducted a prospective cross-sectional consists of 30 low-risk newborn infants at the Hospital Estadual Mário Covas - Santo André (SP). The Dubowitz neonatal neurological examination was administered to newborn infants born with gestational ages between $37^{\text {th }}$ and $42^{\text {th }}$ weeks, birth weight greater than or equal to $2,500 \mathrm{~g}$ without records of events. The results was calculated by mean and standard deviation, and the level of significance was $p<0.05$ for comparison between the scores of the evaluations, the Chi-square. Results: $80 \%$ of term neonates showed expected score for normality $(p<0.001)$. None of the characteristics of the studied population was associated with scores lower than expected. Conclusions: the results suggest the possibility of applying the Dubowitz method in our population due to the similarity of responses.

Keywords: infant, newborn; child development; neurologic examination.

Recebido em: 08/06/2010

Revisado em: 20/05/2011

Aprovado em: 03/10/2011

\footnotetext{
Trabalho realizado no Hospital Estadual Mário Covas (HEMC) - Santo André (SP), Brasil.

1 Curso de Fisioterapia da Faculdade de Medicina do ABC (FMABC) - Santo André (SP), Brasil.

2 Hospital Estadual Mário Covas (HEMC) - Santo André (SP), Brasil.

Endereço para correspondência: Kathrein Koike do Nascimento - Rua Olavo Bilac, 795, apto 61 - Vila Euclides - CEP: 09725-570 -

São Bernardo do Campo (SP). Brasil-E-mail: ka koike@hotmail.com.

Fonte de Financiamento: nenhuma.

Conflito de interesse: nada a declarar.
} 


\section{Introdução}

A avaliação neurológica neonatal é de suma importância para a deteç̧ão de prováveis disfunções do desenvolvimento em um recém-nascido (RN), possibilitando, quando necessário, acesso à intervenção multidisciplinar precoce. Uma vez que os primeiros meses de vida consistem no período de maior adaptabilidade e plasticidade do sistema nervoso ${ }^{1,2}$.

Uma avaliação adequada, mesmo em RNs que não apresentaram intercorrências significativas, é de grande utilidade, pois direciona a determinação da vitalidade, do grau de maturidade e das possíveis repercussões precoces ou tardias de complicações clínicas não determinadas nos períodos pré, peri e pós-natal imediato 3 .

O surgimento de novas técnicas de imagem e de neurofisiologia não diminuiu a importância da avaliação clínica e sim possibilitou sua maior especificidade ${ }^{4}$.

Os estudos têm mostrado que, o uso associado de ambas as formas de avaliação, é mais eficaz na determinação de possíveis lesões neurológicas em RNs, assim como, de provável prognóstico ${ }^{5,6}$.

Nas últimas cinco décadas, diversos protocolos foram desenvolvidos para avaliar o estado neurológico do $\mathrm{RN}^{7,8}$. Todos esses métodos de avaliação neurológica neonatal, propostos ao longo do tempo, avaliam os mesmos aspectos, como o tônus de tronco e membros, os reflexos e a movimentação ativa, que seriam preditivos de alterações neuromotoras. Alguns deles também abordam itens comportamentais ${ }^{9-12}$.

No Brasil, Lefèvre foi o precursor da padronização do exame neurológico do recém-nascido a termo (RNT) normal, junto com os autores André-Thomaz e Precht $\mathrm{l}^{9,10}$. O mesmo Prechtl, juntamente com Amiel-Tison ${ }^{12}$ também estudou as várias respostas neurológicas do RNT e documentou seus desvios de maneira objetiva.

Dubowitz e Dubowitz, em 1981, desenvolveram um método de avaliação neurológica neonatal aplicável tanto em recém-nascido pós-termo RNPT quanto nos RNT. Essa versão foi atualizada e revisada em $1998^{9}$.

Desde então, o método tem sido utilizado tanto na prática clínica quanto na pesquisa científica ${ }^{10,11}$ devido à sua validade e reconhecimento internacional ${ }^{12-14}$. É de fácil aplicabilidade, não sendo necessário muito tempo de treinamento. Apresenta instruções para realização de cada item, podendo ser aplicado por qualquer profissional da saúde com conhecimento na área de neurologia ${ }^{15-18}$.

Entretanto, as respostas e as pontuações esperadas para a normalidade foram estabelecidas por meio da análise do comportamento de RNT ingleses e não há estudos nacionais que certifiquem que as mesmas pontuações possam ser estendidas à nossa população. Assim, a obtenção de dados sobre o perfil de respostas em nossos RNT é de suma importância, pois poderá contribuir para uma análise crítica da aplicabilidade do método em âmbito nacional.

O objetivo do presente estudo foi comparar as respostas obtidas na avaliação neurológica neonatal em RNT de baixo risco para lesões neurológicas com as esperadas para a normalidade propostas pelo método padronizado de Dubowitz.

\section{Casuística e Métodos}

Foi realizado estudo prospectivo transversal, composto por 30 RNT de baixo risco para lesões neurológicas do Hospital Estadual Mário Covas (HEMC) - Santo André (SP), vinculado à Faculdade de Medicina do ABC (FMABC). O período de coleta de dados foi de maio a julho de 2009 .

Definiram-se como critérios de inclusão: idade gestacional ao nascimento entre $37^{\mathrm{a}}$ a $42^{\mathrm{a}}$ semanas, peso superior ou igual a $2.500 \mathrm{~g}$ e ausência de registros em prontuário de intercorrências no período gestacional e parto, e assinatura do Termo de Consentimentos Livre e Esclarecido pelo responsável. E como exclusão: presença de más-formações congênitas e síndromes neurológicas; evidências de infecções congênitas, apgar de $5^{\circ}$ minuto inferior a sete e idade maior que três dias de vida no momento da avaliação. O protocolo de avaliação consistiu de um questionário aplicado às mães e um formulário de pontuação da avaliação neurológica.

O projeto foi aprovado pelo Comitê de Ética em Pesquisa da Faculdade de Medicina do ABC (FMABC), protocolo $n^{\circ}$. 036/2009. As avaliações foram realizadas pela mesma examinadora, não sendo realizado treinamento para habilitação. Os RNT foram avaliados 48 horas após o nascimento. Todos os RNs foram avaliados entre as mamadas, nos estágios quatro e cinco de sono e vigília, conforme preconizado por Brazelton ${ }^{19}$. Após a avaliação, foram coletados: 1- Dados maternos: data do parto, idade, número de gestações, abortos e tipo de parto; 2- Dados gestacionais: uso de fumo, álcool drogas e medicamentos, presença de doenças, realização de pré-natal e número de consultas; 3- Dados do RN: peso e comprimento ao nascimento, idade gestacional, classificação de acordo com o peso esperado para a idade gestacional e apgar do $1^{\circ}$ e $5^{\circ}$ minuto.

O protocolo de avaliação neurológica neonatal aplicado foi o de Dubowitz, que consiste em 34 itens agrupados nas seis dimensões seguintes: tônus (dez itens), tipo de tônus (cinco itens), reflexos (seis itens), movimentos (três itens), sinais anormais (três itens) e comportamento (sete itens). A composição das pontuações é calculada pela soma de todos os itens, sendo que se a resposta obtida em cada item for considerada normal, intermediária ou anormal, atribuem-se as pontuações 1,$0 ; 0,5$ e 0,0 , respectivamente. A faixa de normalidade da pontuação total é entre 30,5 e 34, sendo que, nesse estudo, os RNs que obtiveram pontuação acima de 30,5 foram classificados como típicos, e aqueles que obtiveram pontuação inferior, como atípicos. Já para as dimensões, as pontuações de referências são: tônus entre 9 e 10 , tipo de tônus igual a 5 , reflexos entre 5 e 6 , movimentos iguais a 3 , sinais anormais igual a 3 e comportamento entre 6 e 7.

As pontuações obtidas na avaliação neurológica foram analisadas pela distribuição das frequências e valor numérico. Para os dados coletados pelo questionário, foram calculados média e desvio padrão, assim como, também as frequências. O nível de significância adotado foi $\mathrm{p}<0,05$ para análise comparativa entre as pontuações das avaliações 
obtidas neste estudo e as propostas pelo protocolo original de Dubowitz, calculado pelo Teste Qui-quadrado.

\section{Resultados}

De acordo com os dados coletados, via entrevista direta, a idade média das mães foi de 23,47 $( \pm 4,91)$ e da idade gestacional ao nascimento de $38,93( \pm 0,90)$. Em relação aos tipos de parto, $70 \%$ foram normais; 23,3\% cesáreas e 6,7\% fórceps. As mães realizaram, em média, 8,57 consultas de pré-natal, 3,3\% delas apresentaram aborto anteriormente, sendo que 56,7\% referiram uma gestação, 30\% duas gestações e 13,3\% três gestações. Em relação ao consumo de álcool durante a gestação
13,3\% consumiram, já 3,3\% delas faziam uso de drogas ilícitas e 13,3\% utilizaram tabaco. As doenças referidas durante a gestação foram diabetes mellitus (6,7\%), mioma (3,3\%), distúrbio psicótico (3,3\%), epilepsia (3,3\%); sendo que 3,3\% fizeram uso de gardenal e 3,3\% capoten.

Os RNs apresentaram média de peso ao nascimento de 3134,73 gramas e $( \pm 432,28)$, apgar médio no $1^{\circ}$ de 8,4 e no $5^{\circ}$ de 9,6 .

A distribuição das pontuações obtidas em cada um dos itens da avaliação neurológica foi reunida na Tabela 1. Todas as pontuações foram estatisticamente significantes, quando comparadas ao protocolo original de classificação.

As pontuações de maior frequência em cada um dos itens da avaliação nas diferentes dimensões foram reunidas nas Tabelas 2 e 3 .

Tabela 1: Distribuição das pontuações nos diferentes itens da avaliação neurológica

\begin{tabular}{|c|c|c|c|c|c|c|}
\hline & \multicolumn{2}{|c|}{ Típica } & \multicolumn{2}{|c|}{ Atípica } & \multirow[t]{2}{*}{ Total } & \multirow[t]{2}{*}{ Valor $p$} \\
\hline & Valor & $\%$ & Valor & $\%$ & & \\
\hline \multicolumn{7}{|l|}{ Tônus } \\
\hline Postura & 26 & 86,7 & 4 & 13,3 & 30 & $<0,001$ \\
\hline Recolhimento dos braços & 28 & 93,3 & 2 & 6,7 & 30 & $<0,001$ \\
\hline Tração dos braços & 28 & 93,3 & 2 & 6,7 & 30 & $<0,002$ \\
\hline Tração das pernas & 30 & 100 & - & - & 30 & $<0,012$ \\
\hline Ângulo poplíteo & 30 & 100 & - & - & 30 & $<0,001$ \\
\hline Controle de cabeça (1) & 30 & 100 & - & - & 30 & $<0,001$ \\
\hline Controle de cabeça (2) & 30 & 100 & - & - & 30 & $<0,001$ \\
\hline Intervalo da cabeça & 30 & 100 & - & - & 30 & $<0,001$ \\
\hline Suspensão ventral & 27 & 90 & 3 & 10 & 30 & $<0,000$ \\
\hline \multicolumn{7}{|l|}{ Tipo de tônus } \\
\hline Tônus flexor (1) & 30 & 100 & - & - & 30 & $<0,001$ \\
\hline Tônus flexor (2) & 26 & 86,7 & 4 & 13,3 & 30 & $<0,000$ \\
\hline Tônus extensor da perna & 27 & 90 & 3 & 10 & 30 & $<0,001$ \\
\hline Tônus extensor do pescoço (sentado) & 27 & 90 & 3 & 10 & 30 & $<0,000$ \\
\hline Tônus extensor aumentado & 30 & 100 & - & - & 30 & $<0,000$ \\
\hline \multicolumn{7}{|l|}{ Reflexos } \\
\hline Reflexos & 30 & 100 & - & - & 30 & $<0,001$ \\
\hline Sucção/Mordida & 30 & 100 & - & - & 30 & $<0,001$ \\
\hline Preensão palmar & 30 & 100 & - & - & 30 & $<0,001$ \\
\hline Preensão plantar & 30 & 100 & - & - & 30 & $<0,001$ \\
\hline Reflexo de Moro & 30 & 100 & - & - & 30 & $<0,001$ \\
\hline Plancing (colocação) & 30 & 100 & - & - & 30 & $<0,001$ \\
\hline \multicolumn{7}{|l|}{ Movimentos } \\
\hline Movimentos Espontâneos (Qualidade) & 24 & 80 & 6 & 20 & 30 & $<0,001$ \\
\hline Movimentos Espontâneos (Qualidade) & 30 & 100 & - & - & 30 & $<0,001$ \\
\hline Elevação da cabeça em Prono & 29 & 96,7 & 1 & 3,3 & 30 & $<0,012$ \\
\hline \multicolumn{7}{|l|}{ Sinais Anormais } \\
\hline Posturas Anormais dos dedos dos pés e das mãos & 16 & 53,3 & 14 & 46,7 & 30 & $<0,000$ \\
\hline Tremor & 28 & 93,3 & 2 & 6,7 & 30 & $<0,001$ \\
\hline Susto & 28 & 93,3 & 2 & 6,7 & 30 & $<0,001$ \\
\hline \multicolumn{7}{|l|}{ Comportamento } \\
\hline Movimentos dos olhos & 28 & 93,3 & 2 & 6,7 & 30 & $<0,002$ \\
\hline Orientação Auditiva & 29 & 96,7 & 1 & 3,3 & 30 & $<0,033$ \\
\hline Orientação Visual & 16 & 53,3 & 14 & 46,7 & 30 & $<0,000$ \\
\hline Alerta & 26 & 86,7 & 4 & 13,3 & 30 & $<0,000$ \\
\hline Irritabilidade & 20 & 66,7 & 10 & 33,3 & 30 & $<0,000$ \\
\hline Choro & 27 & 90 & 3 & 10 & 30 & $<0,000$ \\
\hline Consolo & 30 & 100 & - & - & 30 & $<0,001$ \\
\hline
\end{tabular}


Tabela 2: Pontuação de maior frequência em cada item da avaliação nas dimensões tônus, tipo de tônus e reflexos

\begin{tabular}{|c|c|c|c|c|c|c|c|c|}
\hline \multicolumn{2}{|c|}{ Tônus } & \multirow{2}{*}{$\begin{array}{l}\% \\
76,7\end{array}$} & \multicolumn{2}{|c|}{ Tipo de Tônus } & \multirow{2}{*}{$\begin{array}{l}\% \\
56,7\end{array}$} & \multicolumn{2}{|c|}{ Reflexos } & \multirow{2}{*}{$\begin{array}{c}\% \\
90\end{array}$} \\
\hline 1-Postura & $\begin{array}{l}\text { 4-Pernas bem } \\
\text { flexionadas }\end{array}$ & & 11-Tônus flexor (1) & 3-Flexão do braço & & 16-Reflexos & 3-Visível & \\
\hline $\begin{array}{l}\text { 2-Recolhimento } \\
\text { dos braços }\end{array}$ & $\begin{array}{l}\text { 4-Braços flexionam } \\
\text { rapidamente }\end{array}$ & 56,7 & 12-Tônus flexor (2) & $\begin{array}{l}\text { 3-Braços e pernas } \\
\text { flexionadas }\end{array}$ & 83,3 & 17-Sucção/Mordida & 3-Suga forte & 100 \\
\hline $\begin{array}{l}\text { 3-Tração dos } \\
\text { braços }\end{array}$ & $\begin{array}{l}\text { 4-Braços flexiona- } \\
\text { dos aprox. } 100^{\circ}\end{array}$ & 66,7 & $\begin{array}{l}\text { 13-Tônus extensor } \\
\text { da perna }\end{array}$ & 3-Tração da perna & 73,3 & 18-Preensão palmar & $\begin{array}{l}\text { 3-Forte flexão dos } \\
\text { dedos }\end{array}$ & 100 \\
\hline $\begin{array}{l}\text { 4-Recolhimento } \\
\text { das pernas }\end{array}$ & $\begin{array}{l}\text { 4-Flexão rapida e } \\
\text { completa }\end{array}$ & 63,3 & $\begin{array}{l}\text { 14-Tônus } \\
\text { extensor do } \\
\text { pescoço(sentado) }\end{array}$ & $\begin{array}{l}\text { 3-Entensão da } \\
\text { cabeça }\end{array}$ & 90 & 19-Preensão plantar & 3-Artelhos curvam & 100 \\
\hline $\begin{array}{l}\text { 5-Tração das } \\
\text { pernas }\end{array}$ & $\begin{array}{l}\text { 4-Flexão rapida e } \\
\text { completa }\end{array}$ & 70 & $\begin{array}{l}\text { 15-Tônus externsor } \\
\text { aumentado }\end{array}$ & 4-Suspensão ventral & 80 & 20-Reflexo de Moro & 3-Completa abdução & 76,7 \\
\hline 6-Ângulo popliteo & $\begin{array}{l}\text { 4-Aproximadamente } \\
90^{\circ}\end{array}$ & 53,3 & & & & $\begin{array}{l}\text { 21-Plancing (colo- } \\
\text { cação) }\end{array}$ & $\begin{array}{l}\text { 3-Resposta com- } \\
\text { pleta }\end{array}$ & 60 \\
\hline $\begin{array}{l}\text { 7-Controle de } \\
\text { cabeça (1) }\end{array}$ & $\begin{array}{l}\text { 4-Eleva a cabeça } \\
\text { mantem a vertical }\end{array}$ & 76,7 & & & & & & \\
\hline $\begin{array}{l}\text { 8-Controle de } \\
\text { cabeça (2) }\end{array}$ & $\begin{array}{l}\text { 4-Eleva a cabeça } \\
\text { mantém }\end{array}$ & 80 & & & & & & \\
\hline $\begin{array}{l}\text { 9-Intervalo da } \\
\text { cabeça }\end{array}$ & $\begin{array}{l}\text { 4-Eleva a cabeça } \\
\text { na linha }\end{array}$ & 66,7 & & & & & & \\
\hline $\begin{array}{l}\text { 10-Suspensão } \\
\text { ventral }\end{array}$ & $\begin{array}{l}\text { 4-Costas } \\
\text { retas,cabeça } \\
\text { alinhada }\end{array}$ & 50 & & & & & & \\
\hline
\end{tabular}

Tabela 3: Pontuação de maior frequência em cada item da avaliação nas dimensões movimentos, sinais anormais e comportamento

\begin{tabular}{|c|c|c|c|c|c|c|c|c|}
\hline \multicolumn{2}{|c|}{ Movimentos } & $\%$ & \multicolumn{2}{|c|}{ Sinais Anormais } & $\%$ & \multicolumn{2}{|c|}{ Comportamento } & $\%$ \\
\hline $\begin{array}{l}\text { 22-Movimentos } \\
\text { Espontâneos } \\
\text { (Qualidade) }\end{array}$ & $\begin{array}{l}\text { 4-Movimentos } \\
\text { generalizados }\end{array}$ & 80 & $\begin{array}{l}\text { 25-Posturas } \\
\text { Anormais dos } \\
\text { dedos dos pés e } \\
\text { das mãos }\end{array}$ & 4-Adução de polegar & 33,3 & $\begin{array}{l}\text { 28-Movimentos dos } \\
\text { olhos }\end{array}$ & $\begin{array}{l}\text { 3-Movimentos dos } \\
\text { olhos }\end{array}$ & 93,3 \\
\hline $\begin{array}{l}\text { 23-Movimentos } \\
\text { Espontâneos } \\
\text { (Qualidade) }\end{array}$ & $\begin{array}{l}\text { 4-Movimentos } \\
\text { alternados }\end{array}$ & 100 & 26-Tremor & $\begin{array}{l}\text { 3-Tremor só após } 0 \\
\text { Moro }\end{array}$ & 60 & $\begin{array}{l}\text { 29-Orientação } \\
\text { Auditiva }\end{array}$ & $\begin{array}{l}\text { 3-Movimentos dos } \\
\text { olhos }\end{array}$ & 70 \\
\hline \multirow[t]{5}{*}{$\begin{array}{l}\text { 24-Elevação da } \\
\text { cabeça em Prono }\end{array}$} & $\begin{array}{l}\text { 4-Criança eleva a } \\
\text { cabeça }\end{array}$ & 70 & 27-Susto & $\begin{array}{l}\text { 2-Sem sustos } \\
\text { espontâneos }\end{array}$ & 70 & 30-Orientação Visual & 3-Segue horizontalmente & 53,3 \\
\hline & & & & & & 31-Alerta & 4-Quando acordado & 50 \\
\hline & & & & & & 32-Irritabilidade & 2-Acordado chora & 66,7 \\
\hline & & & & & & 33-Choro & 3-Chora ao estímulo & 90 \\
\hline & & & & & & 34-Consolo & 3-Acordado chora & 76,7 \\
\hline
\end{tabular}

A Figura 1 mostra o gráfico da distribuição dos RNs em típicos e atípicos, de acordo com as pontuações totais em cada dimensão.

\section{Discussão}

A similaridade das respostas aqui obtidas, em todos os itens da avaliação, com as propostas pelo protocolo do método Dubowitz foi demonstrada pela correlação significante em todas as pontuações. Porém, na literatura científica foi disponibilizado um pequeno número de estudos que avaliaram RNT saudáveis de acordo com esse método, o que torna a análise comparativa dos dados restrita.

Dentre eles, um foi realizado pelo Dubowitz e Dubowitz ${ }^{7}$, um foi empregado no Vietnã ${ }^{18}$, e outro realizado em nossa população ${ }^{20}$.

O estudo de Dubowitz e Dubowitz $250 \mathrm{RNT}^{7}$ foi realizado para o estabelecimento de uma nova versão do método e aquisição de in- formações mais acuradas sobre as pontuações de cada item. Dos RNs avaliados, $95 \%$ apresentaram pontuação total dentro da esperada para a normalidade, ou seja, considerada ótima. Essa nova versão proposta foi esquematizada em diagramas e, assim, de fácil aplicabilidade ${ }^{\text {. }}$

No presente estudo, $80 \%$ dos RNT apresentaram pontuação esperada para a normalidade. Mas, nenhuma característica da população analisada foi associada às pontuações abaixo do esperado.

No Vietnã, foram analisados 58 RNT de baixo risco sem evidências de intercorrências clínicas, no qual foi aplicada uma versão resumida do método Dubowitz. Em dez dos 25 itens avaliados, a pontuação foi significantemente menor. Os resultados mostraram que em 10 dos 25 itens avaliados, as pontuações obtidas foram significantemente menores, quando comparadas ao estudo original britânico. Embasados nesses dados, os autores sugeriram que a pontuação inglesa de referência não deveria ser usada como padrão-ouro para estudos em outros 
países. Fato que torna necessária a realização de estudos em diferentes países, para uma melhor comparação do desempenho esperado em outras populações ${ }^{18}$.

O único estudo realizado anteriormente no Brasil avaliou uma amostra de RNT saudáveis do mesmo hospital, sem evidências de intercorrências. Foram avaliados 60 RNs e 13,3\% não obtiveram pontuações totais entre 30,5 e 34 . O que difere dos resultados do presente estudo, em que $20 \%$ não atingiram a pontuação acima. Provavelmente, o método necessite de validação nacional para que possa ter valores de maior acurácia para a nossa população ${ }^{20}$.

No atual estudo, a frequência de pontuações totais abaixo do esperado $15 \%$ maior do que a encontrada no trabalho que valida a aplicação do método, pode ter sido influenciada por diversos fatores. Primeiramente, a própria condição nacional de um país subdesenvolvido, com precários níveis sócioeconômicos e culturais, uma vez que o hospital no qual o estudo foi realizado é estadual e atende essencialmente pessoas de baixa renda e também fatores inerentes às diferenças étnicas.

Mesmo os dois estudos nacionais apontando para a necessidade de validação do método para a nossa realidade, o significante número de RNT com pontuações esperadas para a normalidade sugere a possibilidade de aplicação do método Dubowitz em nossa população, uma vez que o propósito essencial de tal avaliação é identificar lactentes com risco de lesão neurológica. Certamente, é preferível encaminhar uma criança para acompanhamento que se desenvolva dentro da

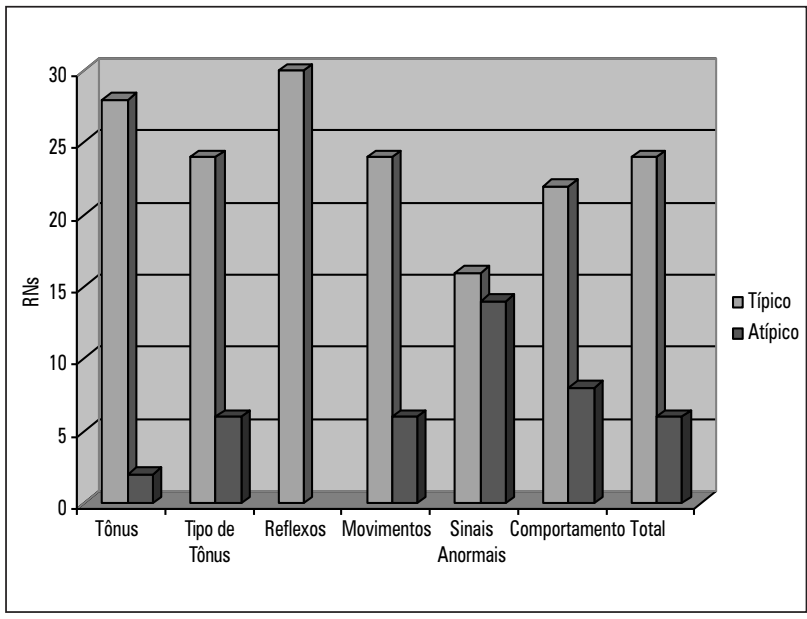

Figura 1: Gráfico das classificações dos RNs de acordo com a pontuação obtida em cada dimensão

normalidade do que não identificar aquelas portadoras de disfunções neurológicas.

Em 2009, um estudo nacional de revisão da literatura científica sobre avaliações padronizadas aplicáveis em RNs e lactentes, reforçou as vantagens do método em relação à sua especificidade, sensibilidade e valor preditivo de disfunções do desenvolvimento ${ }^{21}$.

Perante a escassez de dados nacionais, torna-se clara a necessidade de novos estudos com amostras maiores para possibilitar a adoção do método como instrumento de avaliação para profissionais da saúde envolvidos nos cuidados neonatais em nosso país.

\section{Referências}

1. Vieira MEB, Ribeiro FV, Formiga CKMR. Principais instrumentos de avaliação do desenvolvimento da criança de zero a dois anos de idade. Rev Movimenta. 2009;2(1):23-31.

2. Forslund M, Bjerre I. Neurological assessment of preterm infants at term conceptional age in comparison with normal full-term infants. Early Hum Dev. 1983;195-208

3. Diament A, Cypel S. Avaliação do recém-nascido. In: Neurologia Infantil São Paulo: Ateneu; 2000;783-9.

4. Pedroso FS, Rotta NT. Neurological examination in the healthy term newborn. Arq Neuropsiquiatr. 2003;61(2-A):165-9

5. Dubowitz LMS, Dubowitz V, Palmer MG, Miller G, Fawer CL, Levene MI. Correlation of neurologic assessment in the preterm newborn infant with outcome at 1 year. J Pediatr. 1984;105:452-6.

6. Mercuri E, Guzzetta A, Haataja L, Cowan F, Rutherford M, Counsell S, et al. Neonatal neurological examination in infants with hypoxic Ischaemic encephalopathy: correlation with MRI findings. Neuropediatrics. 1999;30: 83-9.

7. Dubowitz L, Mercuri E, Dubowitz V. An optimally score for the neurologic examination of the term newborn. J Pediatr. 1998;133(3):406-16.

8. Prechtl HFR, Beintema D. The neurological examination of de full term newborn infant. Clin Dev Med. 1964;12:74-106.

9. Mercuri E, Guzzeta A, Laroche S, Ricci D,Vanhaastert I, Simpson A, et al. Neurological examination of preterm infants at term age: comparison with term infants. J Pediatr. 2003:142:647-55.

10. Lefèvre AFB. Contribuição para padronização do exame neurológico do recém-nascido normal. Tese (Dissertação Livre-Docência) - Faculdade de Medicina da Universidade de São Paulo, São Paulo, p. 1950; 32-5.

11. Dubowitz LMS, Dubowitz V. The Neurological assessment of preterm and full term newborn infant. Clin Dev Med. 1981;79(1):103-8.

12. Amiel-Tison C. Neurological evaluation of de maturity of newborn infants. Arch Dis Child. 1968;43:89-93.

13. Molteno C, Gros P, Wallace P, et al. Neurological examination of the preterm and full-term at risk for developmental disabilities using the Dubowitz Neurological Assessment. Early Hum Dev. 1995;41:167-76.

14. Mohsin M, Wong F, Bauman A, et al. Maternal and neonatal factors influencing premature birth and low birth weight in Australia. J Biosoc Sci. 2003:35:161-74 
15. Dubowitz LMS, Levene MI, Morante A, et al. Neurologic signs in neonatal intraventricular hemorrhage: a correlation with real-time ultrasound. J Pediatr. 1981;99(1):127-33.

16. Mcgread R, Simpson J, Panyavudhikrai S, Loo S, Mercuri E, Haataja, L et al. Neonatal neurological testing in resource-poor settings. Ann Trop Paediatr. 2000; 20:323-336.

17. Hieu NT, Gainsboorough M, Simpson JA, Thuy NT, Hang NN, Taylor AM, et al. Neurological status of low-risk vietnamese newborns: a comparison with a british newborn cohort. J Health Popul Nutr. 2006;24(1):57-63.

18. Palmer PG, Dubowtiz LMS, Verghote $M$, et al. Neurological and neurobehavioral differences between preterm infants at term and full-term newborn infants. Neuropediatrics. 1982;13:183-9.
19. Brazelton TB. Neonatal Behavioral Assessment Scale. Clin Dev Med. 1973;50:53-9.

20. Golin MO. Avaliação neurológica neonatal de recém-nascidos pré-termo em idade de termo pelo método Dubowitz comparada a de recém-nascidos a termo. Rev Paul Pediatr. 2009;27(4):402-9.

21. Silva NDSH, Lamy Filho F, Gama MEA, Lamy ZC, Pinheiro AL, Silva DN. Instrumentos de avaliação do desenvolvimento infantil de recémnascidos prematuros. Rev Bras Crescimento Desenvolv Hum. 2011;21(1): 85-98. 